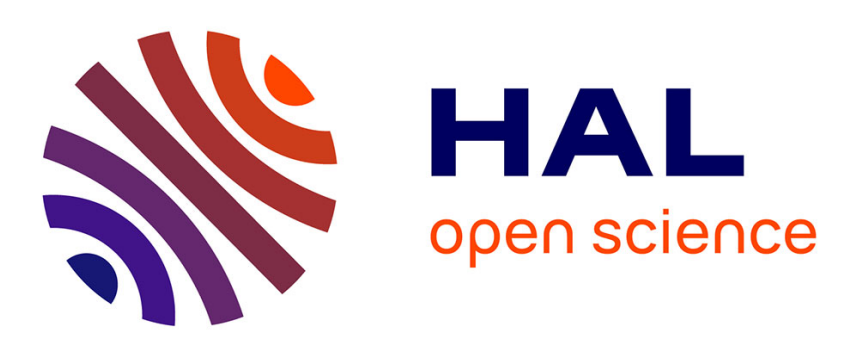

\title{
Automatic multiplanar CT reformatting from trans-axial into left ventricle short-axis view
}

Marta Nuñez-Garcia, Nicolas Cedilnik, Shuman Jia, Maxime Sermesant, Hubert Cochet

\section{- To cite this version:}

Marta Nuñez-Garcia, Nicolas Cedilnik, Shuman Jia, Maxime Sermesant, Hubert Cochet. Automatic multiplanar CT reformatting from trans-axial into left ventricle short-axis view. STACOM 2020 11th International Workshop on Statistical Atlases and Computational Models of the Heart, Oct 2020, Lima, Peru. p.108-116. hal-02961500

\section{HAL Id: hal-02961500 \\ https://hal.inria.fr/hal-02961500}

Submitted on 8 Oct 2020

HAL is a multi-disciplinary open access archive for the deposit and dissemination of scientific research documents, whether they are published or not. The documents may come from teaching and research institutions in France or abroad, or from public or private research centers.
L'archive ouverte pluridisciplinaire HAL, est destinée au dépôt et à la diffusion de documents scientifiques de niveau recherche, publiés ou non, émanant des établissements d'enseignement et de recherche français ou étrangers, des laboratoires publics ou privés. 


\title{
Automatic multiplanar CT reformatting from trans-axial into left ventricle short-axis view
}

\author{
Marta Nuñez-Garcia ${ }^{1,2}$, Nicolas Cedilnik ${ }^{3}$, Shuman $\mathrm{Jia}^{3}$, Maxime Sermesant ${ }^{3}$, \\ and Hubert Cochet $^{1,4}$ \\ 1 Electrophysiology and Heart Modeling Institute (IHU LIRYC), Pessac, France \\ ${ }^{2}$ Université de Bordeaux, Bordeaux, France \\ 3 Inria, Université C'ôte d'Azur, Sophia Antipolis, France \\ ${ }^{4}$ CHU Bordeaux, Department of Cardiovascular and Thoracic Imaging and \\ Department of Cardiac Pacing and Electrophysiology, Pessac, France.
}

\begin{abstract}
The short-axis view defined such that a series of slices are perpendicular to the long-axis of the left ventricle (LV) is one of the most important views in cardiovascular imaging. Raw trans-axial Computed Tomography (CT) images must be often reformatted prior to diagnostic interpretation in short-axis view. The clinical importance of this reformatting requires the process to be accurate and reproducible. It is often performed after manual localization of landmarks on the image (e.g. LV apex, centre of the mitral valve, etc.) being slower and not fully reproducible as compared to automatic approaches. We propose a fast, automatic and reproducible method to reformat CT images from original trans-axial orientation to short-axis view. A deep learning based segmentation method is used to automatically segment the LV endocardium and wall, and the right ventricle epicardium. Surface meshes are then obtained from the corresponding masks and used to automatically detect the shape features needed to find the transformation that locates the cardiac chambers on their standard, mathematically defined, short-axis position. 25 datasets with available manual reformatting performed by experienced cardiac radiologists are used to show that our reformatted images are of equivalent quality.
\end{abstract}

Keywords: Automatic image reformatting · Short-axis view · Deep learning segmentation · Cardiac imaging

\section{Introduction}

Multiplanar reformatting refers to the process of converting imaging data acquired in a certain plane into another plane. Standard imaging planes are useful to determine normal anatomy and function and to investigate anatomic variants or pathologies. The basic cardiac imaging planes include planes oriented with respect to the heart (e.g. horizontal and vertical long-axis and short-axis (SAX)), and planes oriented with respect to the major axes of the body (e.g trans-axial (TA), sagittal, and coronal). Cardiac pathologies are commonly evaluated using cardiac-oriented planes and therefore the position of these planes should 
be prescribed very accurately. In Computational Tomography (CT) imaging, raw TA images are typically reformatted into SAX view (often the interpretation plane) after manual landmark placement[10]. This manual interaction is operator-dependent and time-consuming as compared to automatic methods. Moreover, the definition of LV SAX plane as going from LV base to apex may differ between observers providing different reformatted images and associated metrics. Marchesseau et al. [12] showed the influence on the evaluation of cardiac function of 4 different SAX acquisition protocols (considering the 2 atrioventricular junctions, considering only the left atrioventricular junction, considering the septum, and considering the LV long-axis).

To the best of our knowledge, no method has been proposed for fully automatic CT image reformatting. However, several methods can be found in the literature for automatic landmark detection and subsequent image reformatting in cardiac MRI. For example, Lu et al. [11] proposed landmark detection using LV segmentations; Le et al. [8] used 3D Convolutional Neural Networks to reformat 4D Flow MR images; and Blansit et al. [3] proposed to use U-Net-based heatmap regression to reformat cine steady-state free precession series. While these methods require manual annotation of anatomical landmarks (tedious and time-consuming) Alansary et al. [2] used acquired standard views for training avoiding the need for any manual labeling. The authors employed a multi-scale reinforcement learning agent framework that enables a natural learning paradigm mimicking operators' navigation steps.

Reformatting to SAX view can be done by resampling the raw TA image along its subject-specific LV SAX plane. In this paper, we propose to first segment the left and right ventricles and use the masks to automatically locate a few anatomical features needed to accurately compute the LV SAX plane. As compared to Deep Learning (DL) methods, our approach does not require annotated data or training a model, usually expensive and time-consuming.

\section{Methods}

Let $m=\{\mathrm{XYZ}\}$ be the coordinate system in original TA view and let $m^{\prime}=$ $\left\{X^{\prime} Y^{\prime} Z\right.$ ' $\}$ be the coordinate system in standard SAX view (see Figure 1). The standard SAX view can be defined as follows: the LV long-axis is aligned according to the Z'-axis; and the right ventricle (RV) is positioned on the left side of the LV as seen in the X'Y'-plane. The reformatting to SAX view is computed by resampling the input TA view along its subject-specific LV SAX plane. Image resampling involves 4 components [1]:

1. The image that is sampled, given in coordinate system $m$ (i.e. the input image in TA view).

2. A resampling grid, regular grid of points given in coordinate system $m^{\prime}$.

3. The transformation $T_{m^{\prime}}^{m}$ that maps points from coordinate system $m^{\prime}$ to coordinate system $m$. 


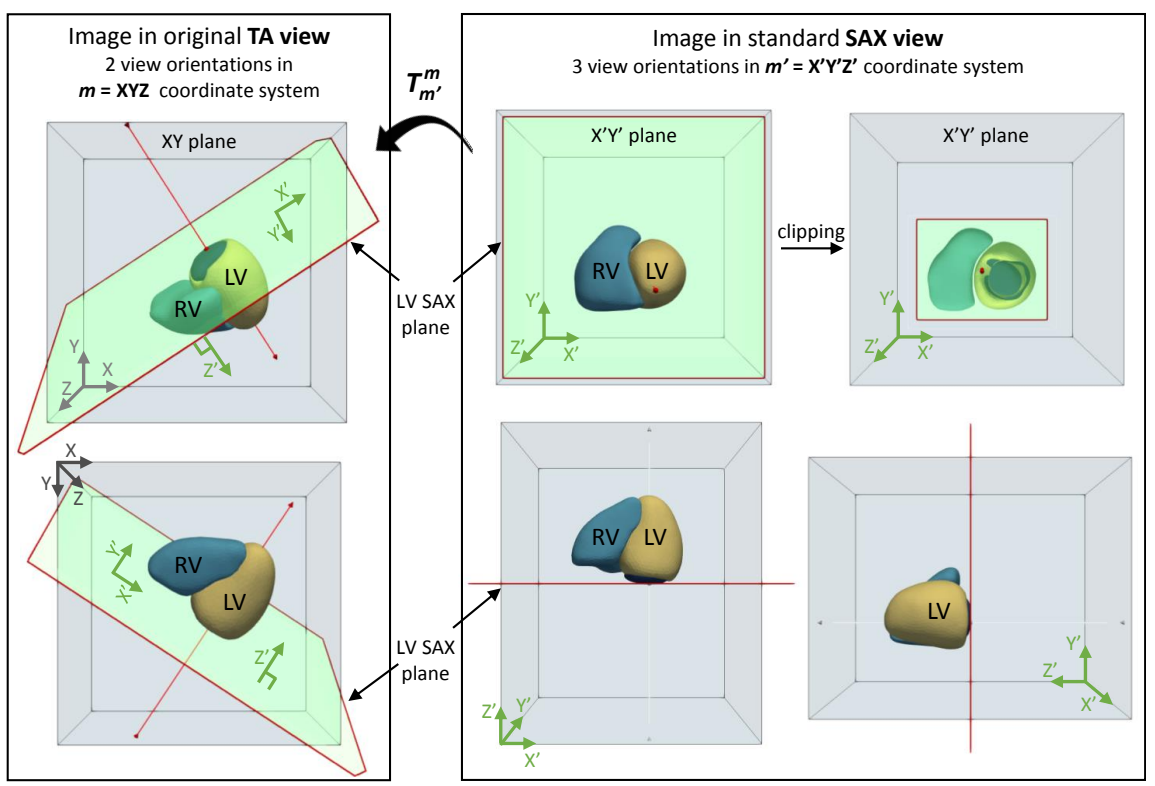

Fig. 1: Schematic representation of multiplanar reformatting to SAX view. On the left, original TA view in $m$ coordinate system. On the right, corresponding SAX view in $m^{\prime}$ coordinate system. For simplicity, only surface models of the LV and RV are shown. $\mathrm{LV}=$ Left Ventricle; RV = Right Ventricle; $\mathrm{TA}=$ Trans-axial; $\mathrm{SAX}=$ Short-axis.

4. An interpolator method for obtaining the intensity values at arbitrary points in coordinate system $m$ from the values of the points defined by the sampled image.

In our approach, cardiac ventricles are first segmented from the original CT image and surface meshes are computed from the corresponding masks (Section 2.1). Mesh coordinates are used to calculate the subject-specific transformation $T_{m^{\prime}}^{m}$ (Section 2.2) that is finally used to resample the input TA image generating its corresponding SAX view (Section 2.3).

\subsection{Image segmentation}

Automatic segmentation of the cardiac ventricles is performed using a DL-based framework. The method, previously described in $[7,4]$ relies on the use of two successive 3D U-nets [13]. The high spatial resolution of CT images requires high memory resource while at the same time the ventricles take only a fraction of the entire CT volume. In our approach, we input to the first network a low-resolution version of the input data. The output (coarse segmentation) is used to locate the ventricles and keep only the region around them. A high-resolution cropped subvolume is input to the second 3D U-net. The resulting segmentations are post- 
processed to obtain clean and non-overlapping masks including up-sampling to the original CT image resolution. The model was trained using $450 \mathrm{CT}$ scans with corresponding expert segmentations of the LV endocardium and epicardium, and the right ventricular epicardium. $50 \mathrm{CT}$ scans were used for validation with a loss function defined as the opposite of the Dice score. Importantly, the trained model was already available to us and we only use it for inference (i.e. prediction or testing) in this work. Therefore, any labeled data or training phase is required for the work presented in this paper.

After image segmentation, triangular surface meshes are computed from the masks using the marching cubes algorithm [9] and they are next uniformly remeshed to improve their quality $[14]^{5}$.

\subsection{Transformation computation}

The transformation $T_{m^{\prime}}^{m}$ that maps points from coordinate system $m^{\prime}$ to coordinate system $m$ can be computed as the inverse of the rotation matrix that rotates points from TA orientation to SAX orientation. Let this rotation matrix be $\boldsymbol{R}_{\boldsymbol{S A X}} \in \mathbb{R}^{3 \times 3}$. It takes into account 3 different rotations that locate 3 different $L V$ features on their target position in SAX view:

1. $\boldsymbol{R}_{M V} \in \mathbb{R}^{3 \times 3}$ aligns the mitral valve (MV) plane.

2. $\boldsymbol{R}_{\boldsymbol{S}} \in \mathbb{R}^{3 \times 3}$ aligns the $\mathrm{LV}$ septum (location of the RV with regard to the $\mathrm{LV}$ ).

3. $\boldsymbol{R}_{\boldsymbol{L} \boldsymbol{A X}} \in \mathbb{R}^{3 \times 3}$ aligns the LV long-axis.

$\boldsymbol{R}_{\boldsymbol{S A X}}$ is computed as the result of the matrix multiplication:

$$
R_{S A X}=R_{L A X} R_{S} R_{M V}
$$

Figure 2 depicts two examples where the alignments are shown consecutively for illustrative purposes only.

All rotation matrices in our approach are computed using the following processing. Given two unit vectors, $\overrightarrow{u_{1}}$ and $\overrightarrow{u_{2}}$, a rotation matrix $\boldsymbol{R}$ that aligns $\overrightarrow{u_{1}}$ to $\overrightarrow{u_{2}}$ can be computed as follows:

$$
\boldsymbol{R}=\boldsymbol{I}_{\mathbf{3}}+\boldsymbol{V}_{\times}+\boldsymbol{V}_{\times} \boldsymbol{V}_{\times} \frac{1-c}{s^{2}}
$$

where,

$\boldsymbol{I}_{\mathbf{3}}=3 \times 3$ Identity matrix

$\vec{v}=\overrightarrow{u_{1}} \times \overrightarrow{u_{2}}$,

$s=\|\vec{v}\|$,

$c=\overrightarrow{u_{1}} \cdot \overrightarrow{u_{2}}$, and

$$
\boldsymbol{V}_{\times} \stackrel{\text { def }}{=}\left[\begin{array}{ccc}
0 & -v_{3} & v_{2} \\
v_{3} & 0 & -v_{1} \\
-v_{2} & v_{1} & 0
\end{array}\right] \text { is the skew-symmetric cross-product matrix of } \vec{v}=
$$
$\left(v_{1}, v_{2}, v_{3}\right)$

\footnotetext{
${ }^{5}$ https://github.com/valette/ACVD
} 


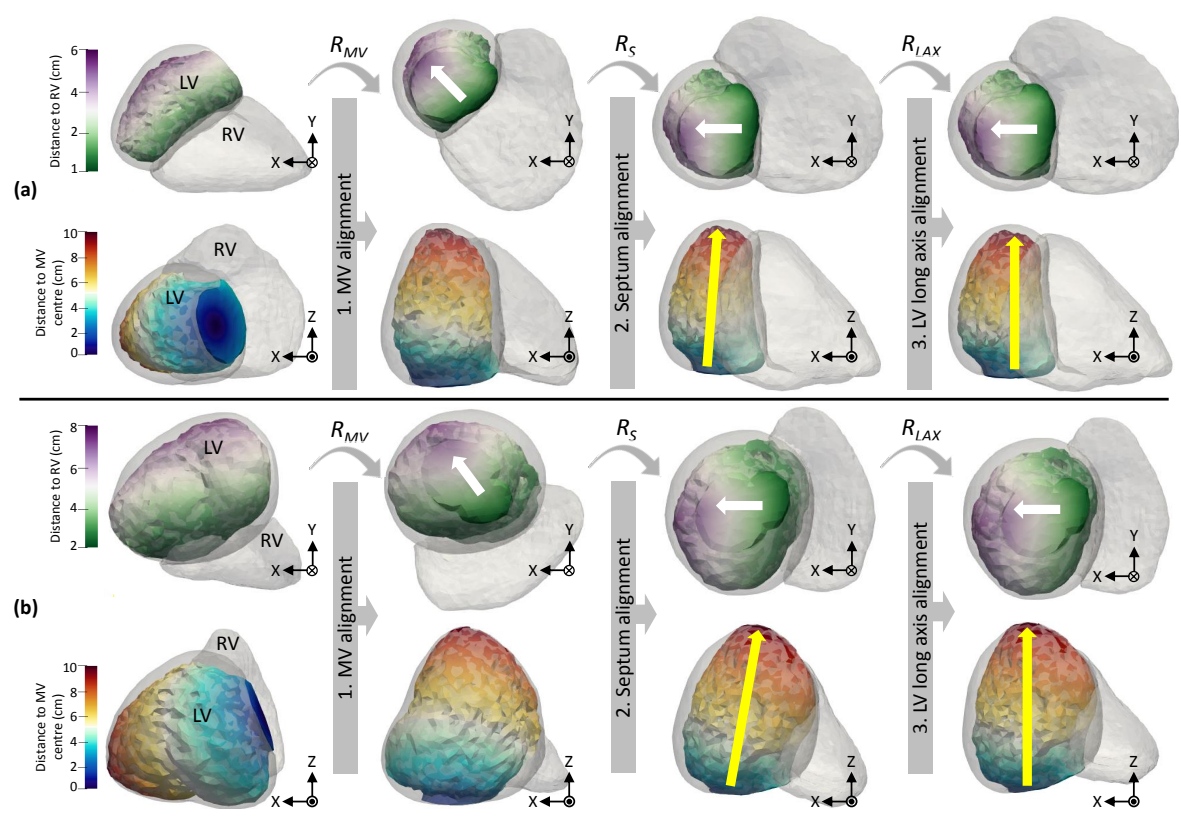

Fig. 2: Two examples of rotation to SAX view using surface meshes. The LV endocardial surface is colored according to its distance to the RV (1st and 3rd row) and according to its distance to the centre of the MV (2nd and 4th row). LV wall and RV epicardial surfaces are shown semitransparent. LV = Left Ventricle; $\mathrm{RV}=$ Right Ventricle; MV = Mitral Valve.

MV plane alignment The rotation matrix $\boldsymbol{R}_{M V}$ is computed using equation 2 where the unit vectors to be aligned are: $\overrightarrow{u_{1}}=$ subject-specific MV normal in TA view; and $\overrightarrow{u_{2}}=\mathrm{MV}$ normal in standard SAX view, i.e. opposite of the X'Y'-plane normal $\left(\overrightarrow{u_{2}}=(0,0,-1)\right)$. To compute $\overrightarrow{u_{1}}$, the region corresponding to the MV orifice is first automatically detected. Distances are computed from each point in the LV endocardial mesh to the LV wall mesh. Similarly, distances are computed from each point in the LV endocardial mesh to the RV epicardial mesh. The MV orifice area is detected as the points in the LV endocardial surface that are far from the LV wall (more than $5 \mathrm{~mm}$ ), and far from the RV epicardial surface. The latter ensures that the MV orifice is not confused with the aortic orifice and it avoids getting orientations corresponding to this artery. The threshold distance was defined to take into account the LV size and was empirically set to more than half the maximum distance between LV endocardium and RV epicardium. All distances are computed using the distance transform $[5,6]$, that assigns to every point in the mesh a value indicating its distance to the nearest point in the other surface. The MV normal $\left(n_{\vec{M} V}\right)$ is computed averaging the normals of all points belonging to the MV orificie area, and $\overrightarrow{u_{1}}$ is defined as the corresponding unitary vector: $\frac{n_{\vec{M} V}}{\left\|n_{\vec{M} V}\right\|}$. 
LV septum alignment The rotation matrix $\boldsymbol{R}_{\boldsymbol{S}}$ is computed using equation 2 where now the unit vectors to be aligned are: $\overrightarrow{u_{1}}=$ unit vector within the MV plane pointing from the RV to the LV; and $\overrightarrow{u_{2}}=$ unit vector within the X'Y'-plane pointing horizontally from RV position to LV position in standard SAX view $\left(\overrightarrow{u_{2}}=(1,0,0)\right)$. White arrows in Figure 2 depict these orientations. To compute $\overrightarrow{u_{1}}$, the surface corresponding to the MV orifice is first extracted using the same procedure explained above. This surface is uniformly remeshed to increase its spatial resolution and distances to the RV are re-computed. Let $p_{0}$ be the point with minimum distance value and let $p_{1}$ be the point with maximum distance value. The unitary vector $\overrightarrow{u_{1}}$ is computed as $\frac{p_{1}-p_{0}}{\left\|p_{1}-p_{0}\right\|}$.

LV long-axis alignment The rotation matrix $\boldsymbol{R}_{\boldsymbol{L} \boldsymbol{A X}}$ is computed using equation 2 where now the unit vectors to be aligned are: $\overrightarrow{u_{1}}=$ subject-specific LV long-axis unit vector; and $\overrightarrow{u_{2}}=$ standard LV long-axis unit vector in SAX view $\left(\overrightarrow{u_{2}}=(0,0,1)\right)$. The LV long-axis is defined as the line connecting the centre of the MV orifice and the LV apex. Yellow arrows in Figure 2 show schematically these orientations. To compute $\overrightarrow{u_{1}}$, the centre of the MV is detected as the centre of mass of the MV orifice surface previously extracted. Let $p_{M V}$ be that point. Distances are computed from $p_{M V}$ to all points in the LV endocardial surface (see Figure 2, 2nd and 4th rows). The LV apex is detected as the point $\left(p_{A}\right)$ that is farthest from $p_{M V}$. Then, $\overrightarrow{u_{1}}$ is computed as $\frac{p_{A}-p_{M V}}{\left\|p_{A}-p_{M V}\right\|}$.

\subsection{Image reformatting}

Prior to image resampling, a reference image is created with desired origin, size, and resolution. The voxels in this reference image determine the resampling grid that is used to sample the input TA image after mapping the voxel's position from $m^{\prime}$ to $m$ using $T_{m^{\prime}}^{m}$. The final transformation also includes a translation to the area where the image to be sampled is located. In our method we propose to use reference_origin $=(0,0,0)$; reference_image_size $=512 \times 512 \times 512$; and reference_image_resolution $=$ input_image_physical_size / reference_image_size.

\section{Results}

The method was applied to 25 images with available reformatting to SAX view performed by expert radiologists. Original CT image dimensions were $512 \times 512$ voxels in the $\mathrm{X}$ and $\mathrm{Y}$ axes and $402 \pm 103$ voxels in the $\mathrm{Z}$-axis. The average original voxel size was $0.40 \pm 0.05 \mathrm{~mm} \times 0.40 \pm 0.05 \mathrm{~mm} \times 0.45 \pm 0.15 \mathrm{~mm}$.

The average time to complete the full image reformatting was $28.38 \pm 7.73$ s (Intel i7 $2.60 \mathrm{GHz} \times 12 \mathrm{CPU}$ and $32 \mathrm{~GB}$ RAM). Several examples of results are shown in Figure 3. It can be seen how our method replicates the manual reformatting and it even outperforms it for some cases. For example in (b), (c), and (d) the LV long-axis is more accurately aligned along the Z-axis using our approach than using manual reformatting. 


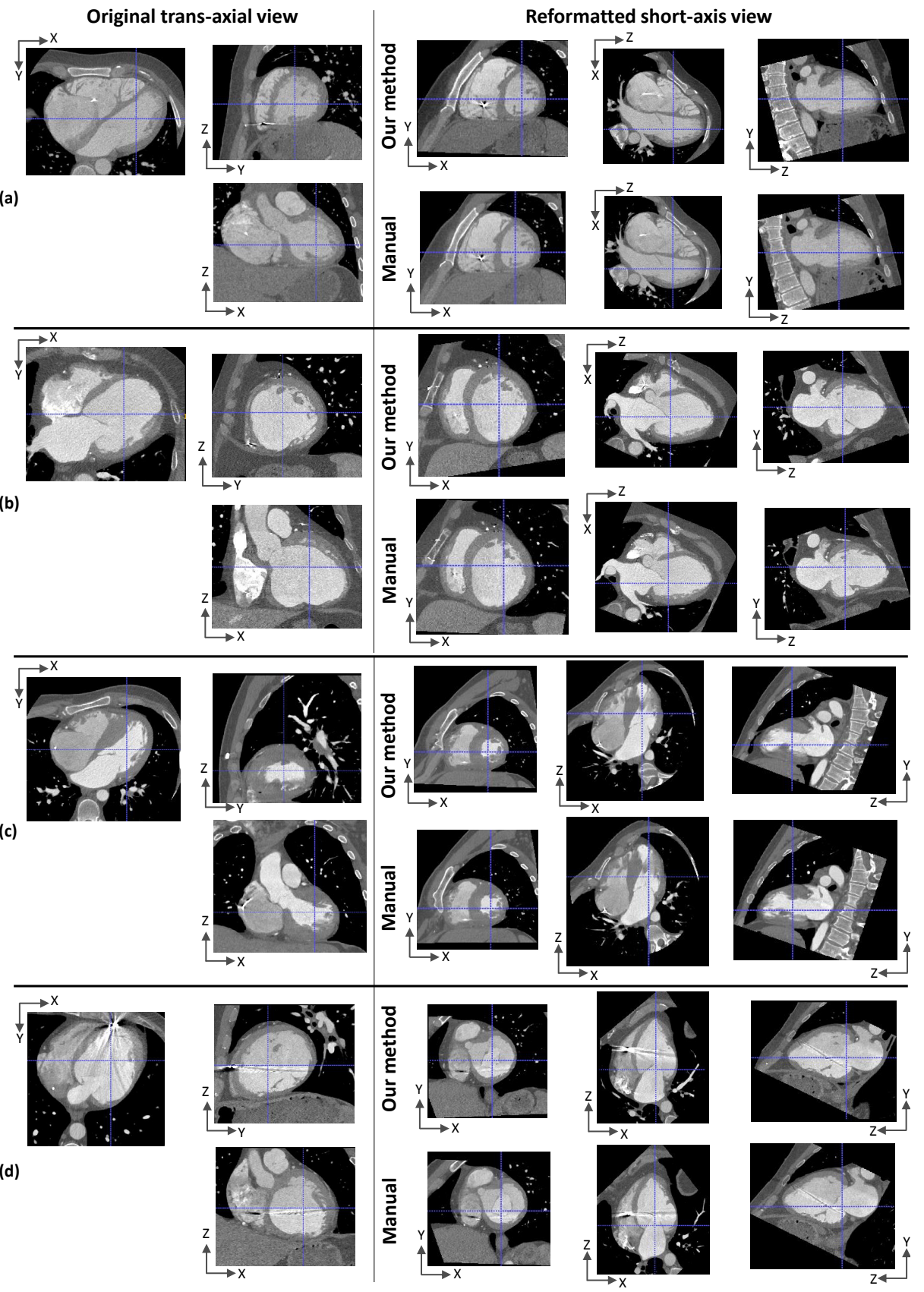

Fig. 3: Example results. On the left, 3 views of original trans-axial images; on the right, 3 views of corresponding short-axis images reformatted with our method (top), and the provided manual reformatting (gold standard) (bottom). 


\section{Conclusions}

Cardiovascular image interpretation often relies on multiplanar reformatted images typically computed manually being time-consuming and lacking reproducibility. Automatic methods such as DL-based techniques often require lots of annotated data and computationally expensive resources. We have presented a method to reformat raw trans-axial CT images to standard SAX view that it is automatic, fast, accurate, reproducible and it does not require labeled data. The main drawback is however the dependency of the method on the quality of the segmentation masks. Previous studies have shown highly precise segmentation results using our proposed method (median Dice score of 0.9 [4]) but the image segmentation step (Section 2.1) could be easily replaced by any desired segmentation method (including manual segmentation performed by a radiologist which is yet considered the gold standard). Computing accurate standard SAX images may be useful to compare different image modalities such as CT and MRI since SAX cardiac MRI is currently one of the most common acquisition views in clinical practice. The origin, size, and resolution, of the reformatted SAX image can be specified by the user which is useful in many applications where a standard representation of the data is required (e.g. to use a pre-trained network).

Acknowledgements. Part of this work was funded by the ERC starting grant EC-STATIC (715093), the IHU LIRYC (ANR-10-IAHU-04), the Equipex MUSIC (ANR-11-EQPX-0030) and the ANR ERACoSysMed SysAFib projects. This work was also supported by the French government, through the 3IA Côte d'Azur Investments in the Future project managed by the National Research Agency (ANR) with the reference number ANR-19-P3IA-0002. We would like to thank all patients who agreed to make available their clinical data for research.

\section{References}

1. Simple ITK, SPIE2019 COURSE, 02 Images and resampling. https://simpleitk. org/SPIE2019_COURSE/02_images_and_resampling.html, accessed: 2020-07-15

2. Alansary, A., Le Folgoc, L., et al.: Automatic view planning with multi-scale deep reinforcement learning agents. In: International Conference on Medical Image Computing and Computer-Assisted Intervention. pp. 277-285 (2018)

3. Blansit, K., Retson, T., Masutani, et al.: Deep Learning-based Prescription of Cardiac MRI Planes. Radiology: Artificial Intelligence 1(6), e180069 (2019)

4. Cedilnik, N., Duchateau, J., Sacher, F., Jaïs, P., Cochet, H., Sermesant, M.: Fully Automated Electrophysiological Model Personalisation Framework from CT Imaging. In: International Conference on Functional Imaging and Modeling of the Heart. pp. 325-333 (2019)

5. Danielsson, P.E.: Euclidean distance mapping. Computer Graphics and image processing 14(3), 227-248 (1980)

6. Fabbri, R., Costa, L.D.F., et al.: 2D Euclidean distance transform algorithms: A comparative survey. ACM Computing Surveys (CSUR) 40(1), 1-44 (2008)

7. Jia, S., Despinasse, A., Wang, Z., Delingette, H., Pennec, X., Jaïs, P., Cochet, H., Sermesant, M.: Automatically segmenting the left atrium from cardiac images using 
successive 3D U-nets and a contour loss. In: International Workshop on Statistical Atlases and Computational Models of the Heart. pp. 221-229 (2018)

8. Le, M., Lieman-Sifry, J., Lau, F., Sall, S., Hsiao, A., Golden, D.: Computationally efficient cardiac views projection using 3D Convolutional Neural Networks. In: Deep Learning in Medical Image Analysis and Multimodal Learning for Clinical Decision Support, pp. 109-116. Springer (2017)

9. Lorensen, W.E., Cline, H.E.: Marching cubes: A high resolution 3D surface construction algorithm. ACM siggraph computer graphics 21(4), 163-169 (1987)

10. Lu, M.T., Ersoy, H., Whitmore, A.G., Lipton, M.J., Rybicki, F.J.: Reformatted four-chamber and short-axis views of the heart using thin section $(\leq 2 \mathrm{~mm}) \mathrm{MDCT}$ images. Academic radiology 14(9), 1108-1112 (2007)

11. Lu, X., Jolly, M.P., Georgescu, B., et al.: Automatic view planning for cardiac MRI acquisition. In: International Conference on Medical Image Computing and Computer-Assisted Intervention. pp. 479-486 (2011)

12. Marchesseau, S., Ho, J.X., Totman, J.J.: Influence of the short-axis cine acquisition protocol on the cardiac function evaluation: a reproducibility study. European journal of radiology open 3, 60-66 (2016)

13. Ronneberger, O., Fischer, P., Brox, T.: U-net: Convolutional networks for biomedical image segmentation. In: International Conference on Medical image computing and computer-assisted intervention. pp. 234-241 (2015)

14. Valette, S., Chassery, J.M., Prost, R.: Generic remeshing of 3D triangular meshes with metric-dependent discrete Voronoi diagrams. IEEE Transactions on Visualization and Computer Graphics 14(2), 369-381 (2008) 